\title{
Connoisseurial contradictions: Expertise modulates faultless disagreement *
}

\author{
Deniz Rudin \\ University of Southern California
}

\author{
Elsi Kaiser \\ University of Southern California
}

\begin{abstract}
We report the results of an experiment investigating faultless disagreement by manipulating the relative expertise of the interlocutors. Our findings show that expertise differences affect judgments of faultless disagreement. We discuss ramifications for prior accounts, and propose a novel account of faultless disagreement in a relativist framework making reference to standards of taste.
\end{abstract}

Keywords: subjectivity, faultless disagreement, relativism, contextualism

\section{Introduction}

This paper is about Faultless Disagreement (FD), the intuition that in arguments like (1), neither party is necessarily 'at fault', i.e., that neither party has made a factual mistake, or that there is some sense in which they can both be 'right'.

(1) A: These buche tacos are delicious.

B: No they're not! These buche tacos are disgusting!

Though this intuition is of a piece with centuries of traditional wisdom (de gustibus non disputandum est), it poses a logical problem for theories of formal semantics (Kölbel 2004): on any standard logic, for any proposition $p$, either $p$ or $\neg p$ must be false. So how can it be that neither party is necessarily incorrect in (1)?

Since Kölbel, it's been a standard assumption that FD is an empirical diagnostic that we can use to binarily sort predicates into two bins: those over which disagreement can be faultless, and those over which it can't. ${ }^{1}$ It has also been a standard assumption that predicates in the former bin, which we'll call Subjective Predicates (SPs), must be assigned an exceptional semantics (Lasersohn 2005; Stephenson 2007; Stojanović 2008; Anand 2009; Pearson 2013; MacFarlane 2014; Coppock 2018, a.o.). The need to explain why SPs display FD has been a prominent motiva-

* Thanks to reviewers and audiences at SALT 31 and CogSci 43 for feedback on this work.

1 Kölbel notes that not all disagreements over such predicates are faultless: for instance, in (1), the intuition of faultlessness disappears if A didn't actually taste the buche tacos. 
tor $^{2}$ of such views, which we follow Anand \& Korotkova (2021) in describing as 'SP-exceptionalist'.

Despite the prominence of FD as a motivator of SP-exceptionalism, the nature of FD as an empirical phenomenon has not received particularly rigorous scrutiny. This paper is part of an ongoing research project seeking to better understand the empirical profile of FD. Recent experimental work challenges the idea that predicates can be categorized into those that exhibit FD and those that don't: Scontras, Degen \& Goodman (2017) show that it may not be possible to binarily bin predicates into those that exhibit FD and those that don't, and Kaiser \& Rudin (2020) provide evidence that a single predicate can give rise to different intermediary degrees of FD as a function of its object. As FD has been a major motivator of SP-exceptionalism, getting a better grip on the empirical signatures of FD is crucial for evaluating the success of the various SP-exceptionialist treatments on the market, and, indeed, may have ramifications for the motivatedness of SP-exceptionalist semantics in the first place.

In the present paper, we explore the role played by the expertise of the interlocutors to further scrutinize the empirical profile of FD. We report an experiment investigating FD, where we varied (i) the relative expertise of the interlocutors involved in the disagreement, and (ii) the domain of taste over which the interlocutors are disagreeing (comparing less-expertise-oriented domains with more-expertiseoriented domains). We also gathered information about participants' attitudes about the legitimacy of expertise in each domain.

Many accounts of FD have sought to attach the intuition of faultlessness to the observation that both speakers can be accurately reporting their own experiences; the disagreement is faultless iff each sentence is true 'as judged by' its utterer (heterogeneously implemented by Lasersohn 2005; Stojanović 2008; Coppock 2018; Zakkou 2019, a.o.). Our experimental manipulation is theoretically relevant because if the relative expertise of the interlocutors affects FD judgments, that cannot be easily explained with reference to the truth of each sentence as judged by its utterer. Moreover, if we find that people's own attitudes about the legitimacy of expertise in a particular domain influence their FD judgments-i.e. if FD is sensitive to features of the assessor that are independent of the context of utterance-this would pose challenges for contextualist accounts.

To preview our findings: we find that FD ratings are indeed affected by the

2 MacFarlane (2014) is notably skeptical of the importance of FD as a motivator for SP-exceptionalism, arguing that "it is not needed for motivating or explaining truth relativism" (p.136). However, MacFarlane also notes that "... the only motivation for positing a coordinate of indices is the presence of an operator that shifts it" (p.60). For evidence that SPs behave identically to other predicates with respect to their shifting behavior, undermining the notion that they are differentially sensitive to a particular index coordinate, see Anand \& Korotkova (2021). 
relative expertise of the interlocutors involved, but only for participants who accept the legitimacy of expertise in the relevant domain (and only in expertise-oriented domains). $\S 3$ gives the details of our experiment, and $\S 4$ summarizes its results. Though we stop short of asserting our findings to be fatal to prior accounts of FD, we outline the problems our findings pose for them in $\S 5$. In $\S 6$, we present a novel positive proposal for a relativist account of FD, extending Coppock's (2018) Outlook-Based Semantics with standards of taste. In $§ 7$, we conclude by discussing the potential for a non-exceptionalist account to explain our results.

\section{Background}

\subsection{Scontras et al. (2017): FD is gradient across predicates}

The literature on SPs has had a tendency to focus on predicates of personal taste (PPTs) like tasty and fun, ${ }^{3}$ and contrasted them with straightforwardly objective predicates. However, 'subjectivity' may be a gradient property of predicates, not a binary property (Fleisher 2013). Empirical support for this idea comes from studies conducted by Scontras et al. (2017), who elicited FD judgments for a large set of predicates, and found gradient intuitions of faultlessness.

Testing disagreements over adjectives classed as describing age (e.g. new), color (e.g. red), material (e.g. wooden), value (e.g. good), shape (e.g. round), dimension (e.g. big), and physical attributes (e.g. smooth), Scontras et al. found no clear binary distinction between 'faultless' and 'faulty' predicates. Disagreements over adjectives of dimension and value were judged highly faultless, age and physical attributes intermediately faultless, and shape, color and material quite faulty, albeit with disagreements over adjective of color somewhat less so than the rest; see Scontras et al.'s Table 1 and Figure 2 for details.

These results suggest that FD is a gradient phenomenon, arising with different predicates to different degrees, rather than binning predicates into those that can display it and those that can't.

\subsection{Kaiser \& Rudin (2020): FD is gradient within predicates}

In previous work, we tested the sensitivity of FD to the identity of the object of predication, with the predicate held constant. In an experiment reported in Kaiser \& Rudin (2020), we elicited FD judgments about disagreements over predicates of gustatory evaluation (tasty, delicious, yucky, disgusting) with objects of three kinds:

3 Though see Anand (2009) for a rich taxonomy of potential SPs, as well as Kölbel (2004) and McNally \& Stojanović (2017) on predicates of aesthetic evaluation, and Crone \& Rudin (2017) on predicates of clarity. 
widely-liked foods (e.g. french fries), divisive foods (e.g. snails), and inedibles (e.g. sand). Disagreements over inedibles were judged dramatically more faulty than the other two categories, and disagreements over widely liked foods were statistically significantly more faulty than divisive foods, despite both falling on the faultless end of the spectrum. These results challenge the view that FD licensing is binary: varying the degree of perceived consensus over the object of predication gives rise to intermediate degrees of FD.

\subsection{Using expertise to investigate FD}

Our prior work found evidence that FD is sensitive to the object of predication. The notion of expertise provides a useful tool to further assess whether judgments of FD are sensitive to factors other than the lexical content of the sentence itself, namely the interlocutors having the disagreement, and the assessor assessing whether a disagreement is faultless or not.

Consider a context like (2), where only one of the speakers is a taco connoisseur. Is the disagreement in (2) as faultless as what we saw in (1)? Or do we defer to the authority of the expert, and consequently view the non-expert's opinion as relatively more 'wrong'? By looking at contexts where the two speakers differ in terms of expertise, we can test whether judgments of FD are sensitive to properties of the interlocutors. ${ }^{4}$

(2) Sam (not an expert about tacos): These buche tacos are delicious.

Taco connoisseur: No they're not! These buche tacos are disgusting!

Furthermore, it could be that someone's judgment of the faultlessness of the disagreement in (2) is modulated not only by the context in which the dialog occurs, but also by that person's own attitudes toward food connoisseurs-i.e., whether someone views connoisseurs' opinions as being privileged, or believes that both experts' and non-experts' opinions are equally valid. By asking about participants' own attitudes, we can address the broader question of whether FD is sensitive to extra-contextual factors, which is relevant to ongoing debates about contextualism and relativism in the semantics of SPs.

\section{Experiment}

\subsection{Design}

Because judgments of FD can be delicate, we conducted an experiment using dialogs like $(3 \mathrm{a}, 3 \mathrm{~b})$ where we manipulated expertise differential (whether the dialog was

4 See Furey (2017) for more on the relation between expertise and FD. 
between two experts or a non-expert and an expert) and the domain of the taste disagreement (wine, beer, art, movies). Here, we provide a summary of our results; see Kaiser \& Rudin (2021) for more details.

a. [two-expert version $]^{5}$

This is a conversation between two wine experts.

One wine expert says: This wine is \{tasty, revolting

The other wine expert says: No, this wine is not $\{$ tasty, revolting $\}$.

b. [one-expert version]

This is a conversation between Andy (who has no expertise in wine) and a wine expert.

Andy says: This wine is \{tasty, revolting

The wine expert says: No, this wine is not $\{$ tasty, revolting $\}$.

In what follows, we use the term EXPERTISE DIFFERENTIAL when describing whether judgments of FD are modulated by whether both speakers are experts. If FD is simply a reflex of the lexical content of a sentence, irrespective of the context of utterance or the index of evaluation, it should show no sensitivity to properties of the interlocutors. However, if the interlocutors' expertise levels modulate participants' judgments of FD—i.e., if we find effects of expertise differential-this would mean that FD cannot be a 'fixed' property of the lexical content of a sentence and instead depends (at least) on contextual information about the interlocutors.

We also test whether judgments of faultless disagreement are modulated by participants' own attitudes about whether expert opinions in a domain are more valid than laypeople's opinions. Such participant attitude effects would be further evidence that faultless disagreement does not follow directly from the lexical content of a sentence. For example, if one of the experimental participants holds the opinions of wine experts in high regard, but another participant thinks that there is no such thing as wine expertise, will they attribute different levels of faultlessness to (3a) and (3b)? If yes, this would indicate that judgments of FD depend not only on the pair of a sentence and a context of utterance, but on assessors' own attitudes about the subjective domain: prima facie evidence against a contextualist account of FD, and in favor of a relativist one.

If judgments of FD are sensitive to the assessor's attitudes about the specific domain, the judgments may vary across domains. 'High-culture' domains—where expert status is established and valued (e.g. wine, art)—-may show stronger expertise differential effects than 'popular culture' domains (e.g. beer, movies) which are

5 Only one adjective was displayed to participants for a given item. Two example adjectives are given in $(3 \mathrm{a}, 3 \mathrm{~b})$ to illustrate that we tested a mix of positive and negative SPs. In one-expert conditions, the non-expert spoke first, to avoid creating a pragmatically odd context where a non-expert 'corrects' an expert. 
viewed as more accessible to untrained consumers. We use the terms more vs. less expertise-oriented to refer to this distinction.

\subsection{Method}

We report data for 84 adult native speakers of U.S. English who participated over the internet. We tested dialogs like (3), constructed using eight positive (e.g. delicious, amazing, inspiring) and eight negative PPTs (e.g. revolting, boring, confusing). ${ }^{6}$ As in (3), a preamble signaled whether the dialog was between two experts or one expert and one non-expert. Each participant saw each subjective predicate only once. Across participants, each gustatory-condition predicate was presented with both beer and wine, and each visual-condition predicate with both movies and art. ${ }^{7}$ Thus, any potential differences between more vs. less expertise-oriented domains cannot be blamed on particular predicates. The study consisted of 16 targets and 24 fillers, presented with a Latin-Square design.

We collected two kinds of data. During the main experiment, we collected ratings of faultless disagreement: people read dialogs like (3) and used a six-point scale to indicate whether they thought both speakers could be right or whether one is wrong (1 was labeled 'One of the two people is wrong' and 6 was labeled 'Both people can be right'). Higher ratings indicate higher faultlessness.

Afterwards, we asked participants about their own attitudes regarding expertise. Participants were asked, for each domain, whether they view expert opinions as more valid than non-expert opinions or whether they think everyone's opinion is equally valid. We also asked participants to self-identify as experts in any of the domains. Because very few did, we do not discuss these data further.

\section{Results}

We first summarize the data regarding participants' attitudes about the legitimacy of expert opinions in the different domains $(\$ 4.1)$. We then present the results from the main experiment about whether judgments of FD are influenced by the expertise of the interlocutors $(\$ 4.3)$ or by the experimental participants' own views about whether expert opinions in a given domain are more valid than laypeople’s (\$4.2). The results are reported in more detail in Kaiser \& Rudin (2021).

6 Participants saw both positive and negative adjectives in both one- and two-expert conditions. Whether the initial sentence used a positive or negative adjective had no clear effect, so we collapse polarity in our discussion.

7 To be precise, items in the art domain were about paintings. 


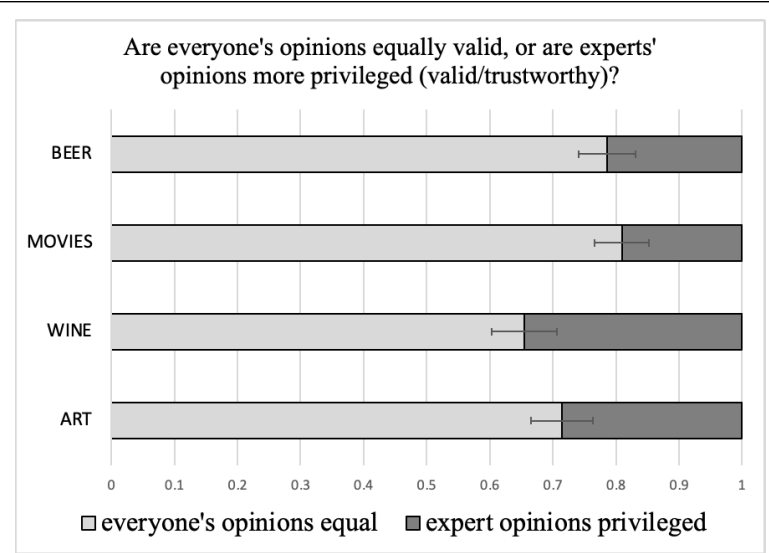

Figure 1 Responses to post-experiment questions about participants' attitudes regarding the validity of expert opinions vs. laypeople's opinions in each domain

\subsection{Differences between domains}

The post-experiment questions about the validity of experts' vs. laypeople's opinions reveal that although there is an overall preference to view everyone's opinions as equally valid, expert opinions are more likely to be viewed as privileged (darker grey) in the domain of wine and art than beer and movies ( $<<.01)$ (Fig. 1). This supports our assumption that the domains of wine and art are more expertise-oriented than beer and movies.

\subsection{Participants' own attitudes influence FD ratings}

Turning now to FD ratings, once we look at each domain as a function of participants' attitudes about the validity of expert vs. laypeople's opinions in the domain, we see clear effects of participant attitudes. Figure 2 shows that in expertise-oriented domains (wine, art), disagreements are rated more faultless (higher bars) by those participants who regard everyone's opinions as equally valid, compared to those who regard expert opinions as privileged (t's $>|2|$ ). I.e., participants' own attitudes about the meaningfulness of expert opinions modulate their FD ratings. Essentially, participants who think that 'everyone is entitled to their own opinion' rate disagreements as more faultless. The two less expertise-oriented domains (beer, movies) show the same trend, although it does not reach significance ( $\mathrm{t}$ 's $<|1.6|$ ). (Following convention, $\mathrm{t}>|2|$ ) is treated as significant.) 


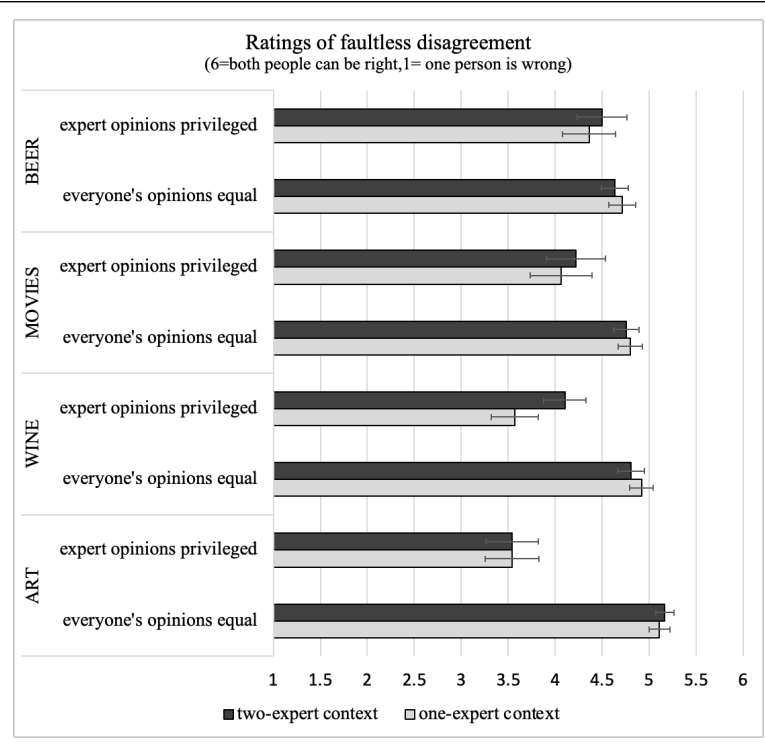

Figure 2 Faultless disagreement (FD) ratings shown as a function of domain and participants' attitudes about expertise in that domain

\subsection{Properties of the interlocutors influence FD ratings}

As can be seen in Figure 2, in the less expertise-oriented domains (beer, movies), ratings of FD are not sensitive to the presence of an expertise differential. Dialogs between two experts (dark grey bars) and dialogs between one expert and one nonexpert (light grey bars) elicit comparable ratings of FD. This is to be expected, given that expertise is not central in these domains.

A different pattern emerges in the domain of wine. Participants' ratings in the one- vs. two-expert conditions pattern differently depending on whether they view experts' opinions as privileged or not (one-expert vs. two-expert $\mathrm{x}$ attitude interaction, $t>|3.7|$ ). In particular, participants who value expert opinions (expertisevaluers) rate one-expert disagreements as more faulty than two-expert disagreements $(t>|2|)$ : when people who value the opinions of wine experts encounter a disagreement between a wine connoisseur and a non-expert, they are more likely to say that one person is wrong, compared to when they are faced with a disagreement between two wine connoisseurs.

Intriguingly, no effects of expertise differential arise in the art domain (light and dark grey bars do not differ). Unlike the domains of beer and movies, where lack of expertise differential effects is associated with overall high rates of faultlessness (people don't care if it's two experts or an expert and a non-expert because everyone's opinion is legitimate), in the art domain art-expertise-valuers feel that all art-related 
Connoisseurial contradictions

disagreements are relatively faulty (short bars).

We return to the asymmetry between the wine and art domains in $\S 6$.

\subsection{Summary}

Our experimental results reveal both expertise-valuing effects and expertise differential effects: FD ratings are influenced by differences in participants' views about the value of expertise in a given domain, as well as properties of the interlocutors. These results show that FD cannot be regarded as a reflex simply of the lexical content of the sentence, or even as a reflex of the pair of a sentence and a context of utterance. Extra-contextual factors influence judgments of FD.

\section{Ramifications for theories of faultless disagreement}

Accounts of FD seek to explain two things: first, why are disagreements with particular kinds of predicates faultless? And second, in what sense are these even disagreements? Our results bear only on the former question. For overviews of contemporary views on the latter question, see MacFarlane (2014, ch.6), Zakkou (2019), and Wyatt (2021).

The crucial outcomes from our experiment that a theory of FD must explain are the following two: (I) FD is sensitive to the relative expertise of the interlocutors (the 'expertise differential effect'), and (II) FD varies with the assessor's beliefs about the legitimacy of expertise in a given domain (the 'expertise-valuing effect'). The former suggests that an explanation of FD in terms of both sentences being true-as-judged-by-their-utterers cannot be the whole story; the latter suggests that a successful account of FD cannot be fully contextualist, as FD is sensitive to features of the assessor that are independent of the context of utterance.

In $\$ 5.1$ we discuss ramifications for contextualist accounts; in $\$ 5.2$ we discuss ramifications for relativist accounts.

\subsection{Problems for contextualist accounts}

The observation that FD is sensitive to factors entirely independent of the context of utterance is prima facie problematic for contextualist accounts of FD. ${ }^{8}$ However, proponents of contextualist semantics for SPs might in principle be happy to offload the explanation of FD to pragmatics (Zakkou 2019). We'll consider how that offloading might work here.

8 See Anand \& Korotkova (2021) for an independent source of evidence against contextualist accounts of SPs. 
The most popular family of contextualist proposals treat SPs (in the absence of overt assessor PPs like to Julie) as generic statements about the tastes or perceptual responses of evaluators (e.g. Anand 2009; Moltmann 2010; Pearson 2013; Muñoz 2019, 2021). ${ }^{9}$ Glossing over the details of generic quantification, on these views a sentence like These buche tacos are delicious is true iff generic perceivers find buche tacos to be delicious. In order to capture facts about the normative force of taste claims, some accounts include a quantificational restriction to NORMAL or otherwise NORMATIVELY QUALIFIED perceivers (e.g. Anand 2009; Crone \& Rudin 2017; Muñoz 2021), or gesture toward a defeasible pragmatic default that the speaker takes their taste to be shared by the other interlocutors (Pearson 2013, q.v. López de Sa's 2008 'Presupposition of Commonality'). In order to capture facts about the speaker-orientedness of taste claims, some accounts include a quantificational restriction to perceivers that the speaker IDENTIFIES WITH (Moltmann 2010; Pearson 2013), or gesture toward a defeasible pragmatic default that the speaker identifies themself with normal/qualified perceivers (e.g. Crone \& Rudin 2017; Muñoz 2021; see also Zakkou's 2019 'Superiority Approach').

Restricting generic quantification to perceivers the speaker identifies with (which we'll refer to as a restriction of 'speaker identification' for convenience) cannot straightforwardly capture the expertise differential effect. For concreteness, consider the semantics put forward by Pearson (2013), where generic quantification is expressed as universal quantification over counterparts in worlds accessible via a generic accessibility relation:

$$
\begin{aligned}
& \llbracket \text { These buche tacos are tasty } \rrbracket^{c, w}=\left[\forall x, w^{\prime} \mid \operatorname{ACC}\left(w, w^{\prime}\right) \wedge\right. \\
& \left.C\left(\text { buche-tacos }, x, w^{\prime}\right) \wedge I(\operatorname{SPEAKER}(c), x)\right] \text { tasty' }\left(\text { buche-tacos }, x, w^{\prime}\right)
\end{aligned}
$$

(adapted with minor modifications from Pearson ex.65c)

In prose: the buche tacos are tasty in $w$ iff at all worlds accessible from $w$ via a generic accessibility relation ACC and for all individuals bearing the identifies-with relation $I$ to the speaker, if that world has a relevant counterpart for that individual and the buche tacos (expressed by the 3-place counterpart relation $C$ ), then that individual finds the buche tacos tasty in that world. Or, to simplify: "everyone I identify with finds these buche tacos tasty in all normal/relevant worlds."

As Pearson notes, in order to make sense of why disputes over taste predicates even count as disagreements on this view, we have to bite the bullet and assume that the interlocutors identify with the same set of individuals, including at least the interlocutors involved in the disagreement. However, this also entails that (at least) one speaker must have said something false, leaving the intuition of faultlessness

9 Some contextualist accounts (e.g. Stojanović 2008; Zakkou 2019) instead treat SPs as covertly indexical, i.e. $\llbracket$ tasty $\rrbracket=\llbracket$ tasty to $m e \rrbracket$. Such accounts will struggle with our observations for the same reason as relativist accounts like Lasersohn (2005); see discussion in §5.2. 
difficult to explain. Pearson suggests that the intuition of faultlessness stems from the intuition that both speakers are justified in generalizing from their personal taste to the tastes of those in their identification set, even though at least one of them must have done so in error. So why would that intuition be weakened when one of the interlocutors is an expert and the other is not? One might claim that sets of individuals including both experts and non-experts are more heterogenous in their tastes than sets of individuals including only experts, making the interlocutors less justified in generalizing from their own experiences. But the claim that experts have more homogenous taste than non-experts is dubious, given the regularity with which experts disagree in subjective domains, and it's not clear why only expertise-valuers would make this assumption.

In fact, one might claim that experts and non-experts are less likely to include each other in their identification sets in the first place: experts and non-experts are less likely to believe their tastes to be shared. But this leads to the incorrect prediction that expertise differential contexts should be interpreted as more faultless. It's simply not clear, in view of speaker identification alone, why an assessor's preference for the tastes of the expert should make any difference for their evaluation of either interlocutor's claim that those who she identifies with share her opinions about the wine.

A contextualist semantics that restricts generic quantification to a normatively defined set of perceivers may have more traction. Consider a minimal modification of (4) in which the predicate of identification $I$ is swapped out for a one-place normativity predicate $N:{ }^{10}$

$$
\begin{aligned}
& \llbracket \text { These buche tacos are tasty } \rrbracket^{c, w}=\left[\forall x, w^{\prime} \mid \operatorname{ACC}\left(w, w^{\prime}\right) \wedge\right. \\
& \left.C\left(\text { buche-tacos }, x, w^{\prime}\right) \wedge N(x)\right] \text { tasty }\left(\text { buche-tacos }, x, w^{\prime}\right)
\end{aligned}
$$

Proponents of such accounts could pursue an explanation of FD comparable to Pearson's: expertise-valuing assessors take experts to be more EPISTEMICALLY JUSTIFIED in making claims about the taste of normatively qualified perceivers-a group that expertise-valuers might take to include experts and exclude non-expertswhereas non-expertise-valuing assessors see all tasters as equally epistemically justified in making claims about the taste of normatively qualified perceivers-a group to which non-expertise-valuers might apply a more egalitarian standard of membership. The intuition of faultlessness could be attached, a la Pearson, to the judgment that both parties are epistemically justified in making their claims, though, given that this is a contextualist account, one or the other of them must, strictly speaking, have said something false (for more discussion of faultlessness by virtue of epistemic justification see MacFarlane 2014 ch.6, and $\$ 5.2$ below).

10 See Anand (2009), Muñoz $(2019 ; 2021)$ for more fully worked-out proposals along these lines. 
Note that for this explanation to make sense, the relevant notion of normativity must be fine-tuned. If the normativity restrictor $N$ simply specifies that the perceivers that SPs make generic claims about are normal perceivers in the sense of perceiving things as most people do, the explanation above cannot go through: after all, supertasters and master sommeliers are decidedly abnormal perceivers in this sense, though expertise-valuers may view them as exceptionally qualified or adept perceivers. If generic quantification is made normative by virtue of a restrictor $N$ that picks out normatively qualified or adept perceivers, such a normative-generic view can capture our observations along the lines sketched above. ${ }^{11}$

However, this discussion skates dangerously close to relativism. If what counts as a normatively qualified perceiver is something that varies assessor by assessor, rather than something that is settled by the context of utterance, one begins to worry that this purportedly contextualist explanation has simply pushed its relativist character into a black box that it does not explicitly formalize. It remains unclear that a genuinely and thoroughgoingly contextualist account can handle our observations.

\subsection{Problems for relativist accounts}

The most common relativist accounts of SPs are those we could call 'judgedependent' accounts (Lasersohn 2005; MacFarlane 2014). ${ }^{12}$ On these accounts, the truth of sentences is relativized to an individual, called a 'judge', which is a coordinate of an index of evaluation in addition to the more familiar coordinates of a world and a time. A sentence like These buche tacos are tasty is true relative to a world, time, and judge iff that judge finds these buche tacos tasty in that world and at that time.

Lasersohn (2005) proposes that a disagreement is faultless iff both statements are true-as-judged-by-their-utterers (what MacFarlane 2014 calls faultlessness by way of 'accuracy'). This conception of faultless disagreement is incompatible with our observations: in expertise differential contexts, both interlocutors have accurately reported their own gustatory responses, and yet expertise-valuers still find them to be significantly less faultless than disagreements in non-expertise differential contexts. The sensitivity of FD to factors other than the accurate reporting of each interlocutor's tastes rules out this explanation of faultlessness.

MacFarlane (2014, ch.6) points out that there are a variety of other ways in which we could understand faultlessness in addition to Lasersohn's. He highlights three

11 Q.v. discussion in Zakkou (2019), who argues that assertions of taste predicates communicate that the speaker takes her standard of taste to be the best standard of taste.

12 What distinguishes Lasersohn's (2005) account from MacFarlane's (2014) is that Lasersohn assumes that the judge of a sentence is the agent of the context of utterance, whereas MacFarlane assumes that it is the agent of the context of assessment. This distinction will not be important for our purposes. 
other possible interpretations of faultlessness: faultlessness by way of the norms of assertion, faultlessness by way of epistemic justification, and faultlessness by way of truth at a fixed point of evaluation. We argue that none of these three other notions of faultlessness can straightforwardly allow a relativist to explain our observations.

If intuitions about FD were explained in terms of whether or not both interlocutors have followed the norms of assertion, the expertise differential effect would then represent (at least) one of the interlocutors being judged to have violated the norms of assertion by virtue of their utterance. One might say, for instance, that expertise-valuers assume a norm of assertion that rules out non-experts making assertions about taste. However, this explanation would predict that expertise-valuers would judge any assertion about taste by a non-expert in any context to violate the norm of assertion, and therefore would incorrectly fail to predict that disagreements among laypeople would be judged relatively faultless. ${ }^{13}$

When it comes to faultlessness with respect to epistemic justification, it's not clear in what way epistemic justification could differentiate taste claims in a judgedependent relativist account that lacks an explicit normative component (see discussion in §5.1). That is to say: if it is indeed true that these buche tacos are tasty as judged by the speaker, then they are epistemically justified in saying so, as it is true in their context of evaluation, regardless of whether it is true in any other context of evaluation. One might object to the notion that truth-in-the-utterer'scontext-of-evaluation is sufficient epistemic justification for assertion. We could take a more sophisticated view of epistemic justification: for example, assertions of taste claims have been analyzed as proposing to coordinate on a judgment shared by all interlocutors (e.g. Stephenson 2007; Furey 2017; Coppock 2018; Rudin \& Beltrama 2019). Though a speaker may be epistemically justified in their belief in the truth of the sentence-as-evaluated-by-themself, they are not necessarily thereby epistemically justified in their belief in the truth of the sentence-as-evaluated-by-all-interlocutors, which is what they have proposed to coordinate on. Such accounts therefore open up the possibility for assertions of SPs to fail to be epistemically justified (i.e. fail to be faultless) if the speaker does not have grounds to generalize from their personal opinion to the opinions of their interlocutors. This is a variation on the explanation in terms of speaker identification discussed in $\$ 5.1$, and it's subject to the same concern: why would the relative expertise of the interlocutors affect either interlocutor's epistemic justification in believing that the other interlocutor agrees with them?

The third way of thinking about faultlessness that MacFarlane highlights is

13 Note that Willer \& Kennedy (2020) propose a special assertive update for SPs, which they take to express an 'experiential grounding' for the asserted proposition. This view does not obviously provide more traction for a norm-of-assertion account of the expertise differential effect: presumably anybody who has had the relevant kind of experience has met the norms for making an experientially grounded assertion. 
faultlessness by way of both sentences being true at a fixed point of evaluation. He dismisses this conception as incoherent: by definition, any index settles the truth of a sentence one way or another; there is no index relative to which any sentence is both true and false (or neither). However, Coppock (2018) proposes a comparable notion of faultlessness in a somewhat different relativist system. In Coppock's account, rather than being relativized to an individual, truth is relativized to what is called an OUTLOOK. On this view, worlds settle only the truth of matters of fact, leaving matters of opinion unsettled. Each world corresponds to a set of outlooks, or possible refinements of it that settle the truth of all matters of opinion as well as matters of fact. Coppock proposes that faultlessness is about truth-at-a-world, not truth-at-an-outlook: it's possible (indeed, necessary) for neither sentence involved in a disagreement over a taste claim to be false at the fixed point of evaluation of a world, because worlds do not settle the truth of taste claims one way or another. But this notion of faultlessness is too strong to capture the expertise differential effect: as Coppock herself notes, this notion of faultlessness predicts that all disputes over taste claims should always be faultless.

In sum, prior accounts of faultlessness couched in both judge-dependent relativism and outlook-based relativism struggle to capture our experimental results. In $\S 6$ we outline a novel relativist proposal for faultlessness that we believe can capture the expertise differential effect.

\section{A positive proposal: Outlooks + standards of taste}

In this section, we develop an analysis of FD designed to capture the expertise differential effect, extending Coppock's (2018) Outlook-Based Semantics with standards of taste. An outlook $o$ is a refinement of a world $w(o \propto w)$ iff it settles the truth of all matters of opinion left open by $w$ while preserving the truth of all factual matters settled by $w$.

(6) Given the set of all possible worlds $W, O$ is the set of all possible outlooks

$$
O_{W}=\{o \mid \exists w \in W \text { s.t. } w \propto o\}
$$

In Outlook-Based Semantics, the index coordinate typically represented by a world in a standard intensional semantics is replaced by an outlook, resulting in relativization of truth to an index of evaluation comprised of an outlook and a time. ${ }^{14}$

14 Crucially, unlike in judge-dependent relativism, an outlook does not stand in for any particular individual assessor: an assessor who is not particularly opinionated might have tastes compatible with a variety of possible outlooks, and it's possible in principle that there are outlooks combining outlandish combinations of opinions that correspond to the taste of no actual assessor. For arguments in favor of outlook-based relativism over judge-based relativism, see Coppock (2018). 
Connoisseurial contradictions

We propose an extension of this model which adds an additional index coordinate: a STANDARD OF TASTE $s$ :

$$
\llbracket \cdot \rrbracket^{i} \quad i=\left\langle t_{i}, o_{i}, s_{i}\right\rangle
$$

A standard of taste $s$ is simply a set of outlooks: it serves to filter out opinions that are not admissible by that standard of taste.

Given $W, S$ is the set of all possible standards of taste

$$
S=\left\{s \subseteq O_{W} \mid \forall w \in W, \exists o \in s \text { s.t. } o \propto w\right\}
$$

A standard of taste may only rule out outlooks from consideration if in doing so it does not also remove worlds from consideration - after all, a standard of taste shouldn't interfere with matters of objective fact.

Standards of taste factor into the evaluation of truth by ruling out all outlooks that are not members of that standard of taste:

$$
\text { For any proposition } p, \llbracket p \rrbracket^{i}=1 \text { iff } o_{i} \in s_{i} \wedge p(o)=1
$$

If a sentence isn't compatible with the standard of taste against which it is being evaluated, that renders it false at all possible outlooks.

Here's a concrete example: the NorCal Mexican Food Snob standard of taste $s_{\text {mex }}$ contains no outlooks at which what is served by Taco Bell is tasty; contains outlooks at which what is served by Cholita Linda is tasty, and outlooks at which it is not tasty; and contains no outlooks at which what is served by La Taqueria is not tasty. For ease of reference, we'll refer to outlooks that are members of a standard of taste as PERMISSIBLE or LEGITIMATE relative to that standard of taste. Relative to $s_{\text {mex }}$, some pro-Cholita Linda outlooks are permissible, as are some anti-Cholita Linda outlooks (provided that neither are also pro-Taco Bell or anti-La Taqueria). That is to say, heterogeneity of opinion about Cholita Linda is acknowledged as legitimate by this standard of taste: it is possible for Cholita Linda to be either tasty or not tasty (at different outlooks). However, heterogeneity of opinion about Taco Bell and La Taqueria is not legitimate relative to this standard of taste: it is impossible for Taco Bell to be tasty or for La Taqueria not to be tasty, regardless of outlook. ${ }^{15}$

Standards of taste allow us to define a novel notion of taste-relative faultlessness:

15 Outside of this footnote, we will set aside the question of the role that standards of taste play in Common Ground update, as what matters for our purposes is only the assessment of a disagreement relative to a standard of taste. Presumably, a Common Ground is a set of outlooks permissible by some standard of taste of some interlocutor in the conversation (i.e. $\bigcup\left\{s_{I} 1, s_{I} 2, \ldots\right\}$ for all interlocutors $\left.I_{1}, I_{2}, \ldots\right)$. If the Common Ground were a set of outlooks permissible by all interlocutors' standards of taste (i.e. $\bigcap\left\{s_{I} 1, s_{I} 2, \ldots\right\}$ ), that would rule out the possibility of ever having Common Ground about anything if two interlocutors' standards of taste are non-overlapping, which seems very very wrong. 
TASTE-RELATIVE FAULTLESSNESS

Assessed relative to standard of taste $s$, disagreement over $p$ is faultless iff $^{16}$ $\exists w, \exists o, o^{\prime} \propto w, \llbracket p \rrbracket^{\langle o, s\rangle}=1 \wedge \llbracket p \rrbracket^{\left\langle o^{\prime}, s\right\rangle}=0$

That is to say: a disagreement is judged faultless by the bearer of a standard of taste iff that standard of taste allows for outlooks validating both sides of the argument to be refinements of the same world. ${ }^{17}$ Note that on this view, only disagreements involving SPs can be faultless, because only SPs can have truth values that vary across outlooks that are refinements of the same world. For objective propositions, which receive the same truth value across all refinements of any given world, there can be no pair of such refinements at which the truth value of the proposition differs, be definition. But it is no longer the case that all disagreements involving SPs are faultless - this is only true relative to standards of taste that permit either opinion.

Coming back to the expertise differential effect: we can capture this effect by taking expertise-valuers to be unwilling to adopt standards of taste that allow for divergence of opinion from the tastes of experts: ${ }^{18}$ upon observing an expert's judgment, an expertise-valuer adopts a standard of taste that excludes outlooks validating other opinions. This approach leads us to expect that an expertise-valuer will adopt a standard of taste allowing for heterogeneous opinions only if no expert has weighed in yet, or if there is attested disagreement among experts. This fits with the FD judgments of expertise-valuers in our experimental results in the wine domain.

Recall that in the domain of art, we observed that expertise-valuers judge all disagreements about taste to be relatively faulty, regardless of expertise. One might imagine a particularly extreme sort of standard-holder: one who is unwilling to adopt standards of taste that allow for heterogeneity of opinion on a particular subject, period. Call such a standard-holder an OBJECTIVIZER. An objectivizer-about-art is unwilling to adopt a standard of taste that allows for multiple possible truth values for any art-related proposition. Our data suggests that many expertise-valuers in

16 We suppress reference to times here for readability.

17 Though we've kept it from the main text for the sake of simplicity, it would probably be a good idea to restrict quantification over worlds relative to the bearer's epistemic state: if the bearer's standard of taste only allows for heterogeneity of opinion in worlds ruled out by the bearer's knowledge, e.g. in worlds where the wine under discussion has different properties than the bearer knows it to have, then they will not judge the disagreement to be faultless, as all worlds that are allowed by their epistemic state allow for only one opinion about the wine.

18 Note that expertise-valuing standards of taste are just one class of standards of taste among many: the formal apparatus here can be used just as easily to model unsnobbish standards of taste that are unaffected by expert opinions, or even antisnobbish standards of taste that militate against expert opinions. What we present is an account of the effects that standards of taste have on judgments of FD; an account of why people adopt the standards of taste that they do, in our view, is squarely extrasemantic. 
the art domain are such objectivizers: they are unwilling to accept the legitimacy of heterogeneity of opinion even in the case of expert disagreement.

\section{Beyond SP-exceptionalism}

In $\S 5$, we discussed ramification of the expertise differential effect for previous accounts of FD, and in $\S 6$, we proposed a novel account of FD to capture it. However, $\S 1$ noted previous work showing that FD is gradient both across and within predicates, subject to factors other than interlocutor expertise, and the experimental data presented in $\S 4$ adds to this body of evidence. To the extent that a binary notion of FD motivates SP-exceptionalism, the growing body of evidence suggesting that FD is gradient undermines SP-exceptionalism; gradient FD is not straightforwardly captured by any account discussed in $\S 5$, or by our positive proposal in $\S 6$. However, Rudin \& Beltrama $(2019, \S 6)$ sketch a non-exceptionalist view of SPs relying on an internalist interpretation of model-theoretic semantics, and propose an 'interannotator agreement' account of FD. We summarize that account below, and then explore how it relates to our experimental results.

It is a fact about the world that people disagree about the truth of certain statements despite having done the correct empirical probe to assess their truth-in the case of taste predicates, having tasted what is being discussed. The inter-annotator agreement account of FD (IAA) is that FD judgments reflect nothing more than the fact that we have observed many such disagreements (e.g. people disagreeing about what's tasty), and so we are willing to assign a high probability to the disagreement at hand being yet another one of those. On this view, intuitions of faultlessness are not directly reflective of logical properties of the formal semantics of the predicates involved; they reflect merely the observation that those predicates generate low interannotator agreement. That is to say, IAA is a faultlessness-via-epistemic-justification account, but one that does not rely on exceptional semantic machinery for SPs-for an internalist, there is no logical conundrum in the fact that two people's mental representations of the world might diverge despite exposure to the same stimuli.

In this section, we briefly touch on how IAA might deal with the expertise differential effect (\$7.1). We also discuss how it might deal with Kaiser \& Rudin’s (2020) observations about gradient effects of the choice of object on FD, and Scontras et al.'s (2017) observations about gradient judgments of FD across different classes of predicates $(\$ 7.2)$.

\subsection{Inter-annotator agreement and expertise}

Because of the role played by the assumption that both parties have performed the right empirical probe in licensing the intuition of faultlessness, a disagreement might 
be judged faulty even in an area with low inter-annotator agreement if it's not the case that both parties have actually conducted that empirical probe (Kölbel 2004, see fn. 1 above). More relevantly for us, an expertise-valuer additionally might not acknowledge that both parties have actually done the right thing to assess the truth of the sentence: an expertise-valuer either might take only expertly trained perception to be an admissible empirical probe for tastiness, or might take only expert training to qualify someone to accurately assess their own sensory experiences. That is to say, an expertise-valuer might maintain that only an expert is capable of doing the right thing to evaluate the truth of a subjective predicate in a particular domain. The role played by the assumption that both parties have 'done the right thing' allows IAA to explain expertise differential effects.

\subsection{Inter-annotator agreement and gradience}

Kaiser \& Rudin (2020) observe that disagreements over the tastiness of divisive foods are judged more faultless than disagreements over widely-liked foods, which are themselves judged more faultless than disagreements over inedibles $(\S 2.2)$. This is entirely expected given IAA, which crucially predicts an inverse correlation between degree of inter-annotator agreement and degree of faultlessness.

IAA also makes predictions about gradience in FD judgments across predicates (Scontras et al. 2017), though empirical work remains to be done to see whether those predictions are correct. IAA predicts that the degree of FD associated with a class of predicates will be correlated with the likelihood that people with all the relevant facts in hand will agree over the applicability of predicates in that class. Predicates of personal taste are an extreme case of low inter-annotator agreement, such that people frequently disagree even about comparatives. However, low inter-annotator agreement isn't the exclusive provenance of taste predicates: two reasonable people with all the facts in hand could nonetheless disagree over whether somebody counts as tall. ${ }^{19}$ Presumably, multidimensional predicates display lower inter-annotator agreement than monodimensional predicates, ${ }^{20}$ and vague predicates display lower inter-annotator agreement than non-vague predicates, and so on. IAA predicts that gradient inter-annotator agreement translates into gradient judgments of FD. We leave further investigation of the generality of the correlation between inter-annotator agreement and FD, on which the viability of Rudin \& Beltrama's account rests, to future work.

19 Discussion in this paragraph is very much indebted to Fleisher (2013).

20 For more on multidimensionals see Sassoon (2013); Kaiser \& Herron Lee (2017); Solt (2018). 
Connoisseurial contradictions

\section{References}

Anand, Pranav. 2009. Kinds of taste. Manuscript, UC Santa Cruz.

Anand, Pranav \& Natasha Korotkova. 2021. How to theorize about subjective language: a lesson from 'de re'. Linguistics and Philosophy doi:10.1007/s10988021-09331-0.

Coppock, Elizabeth. 2018. Outlook-based semantics. Journal of Semantics 41. 125-164. doi:10.1007/s10988-017-9222-y.

Crone, Phil \& Deniz Rudin. 2017. Assessor-relativizable predicates. Talk presented at Subjectivity in Language and Thought. Handout available: https://denizrudin.github.io/papers/crone_rudin_arp_handout.pdf.

Fleisher, Nicholas. 2013. The dynamics of subjectivity. In Semantics and Linguistic Theory (SALT) 23, 276-294. doi:10.3765/salt.v23i0.2679.

Furey, Heidi. 2017. The paradox of gustatory taste. Ergo 4(17). 481-527. doi:10.3998/ergo.12405314.0004.017.

Kaiser, Elsi \& Jamie Herron Lee. 2017. Predicates of personal taste and multidimensional adjectives: An experimental investigation. In West Coast Conference on Formal Linguistics (WCCFL) 35, 224-231.

Kaiser, Elsi \& Deniz Rudin. 2020. When faultless disagreement is not so faultless: What widely-held opinions can tell us about subjective adjectives. In Linguistic Society of America (LSA) 5, 698-707. doi:10.3765/plsa.v5i1.4757.

Kaiser, Elsi \& Deniz Rudin. 2021. Arguing with experts: Subjective disagreements on matters of taste. In CogSci 43, 924-930.

Kölbel, Max. 2004. Faultless disagreement. Proceedings of the Aristotelian Society 104. 53-73. doi:10.1111/j.0066-7373.2004.00081.x.

Lasersohn, Peter. 2005. Context dependence, disagreement, and predicates of personal taste. Linguistics and Philosophy 28. 643-686. doi:10.1007/s10988005-0596-x.

López de Sa, Dan. 2008. Presuppositions of commonality: an indexical relativist account of disagreement. In Manuel García-Carpintero \& Max Kölbel (eds.), Relative Truth, 297-310. Oxford University Press.

MacFarlane, John. 2014. Assessment Sensitivity. Oxford University Press.

McNally, Louise \& Isidora Stojanović. 2017. Aesthetic adjectives. In James Young (ed.), The Semantics of Aesthetic Judgment, Oxford University Press.

Moltmann, Friderike. 2010. Generalizing detached self-reference and the semantics of generic one. Mind and Language 25. 440-473. doi:10.1111/j.14680017.2010.01397.x.

Muñoz, Patrick. 2019. On tongues: The grammar of experiential evaluation: University of Chicago PhD dissertation.

Muñoz, Patrick. 2021. Acquaintance inferences as evidential effects. Manuscript, 
University of Chicago.

Pearson, Hazel. 2013. A judge-free semantics for predicates of personal taste. Journal of Semantics 30. 103-154. doi:10.1093/jos/ffs001.

Rudin, Deniz \& Andrea Beltrama. 2019. Default agreement with subjective assertions. In Katherine Blake \& Forrest Davis (eds.), Semantics and Linguistic Theory (SALT) 29, 82-102. doi:10.3765/salt.v29i0.4597.

Sassoon, Galid. 2013. A typology of multidimensional adjectives. Journal of Semantics 30. 335-380. doi:10.1093/jos/ffs012.

Scontras, Greg, Judith Degen \& Noah D. Goodman. 2017. Subjectivity predicts adjective ordering preferences. Open Mind 1(1). 53-65. doi:10.1162/OPMI_a_00005.

Solt, Stephanie. 2018. Multidimensionality, subjectivity and scales: Experimental evidence. In Elena Castroviejo, Louise McNally \& Galit Sassoon (eds.), The Semantics of Gradability, Vagueness, and Scale Structure, 59-91. Springer.

Stephenson, Tamina. 2007. Judge dependence, epistemic modals, and predicates of personal taste. Linguistics and Philosophy 30. 487-525. doi:10.1007/s10988008-9023-4.

Stojanović, Isidora. 2008. Talking about taste: disagreement, implicit arguments, and relative truth. Linguistics and Philosophy 30. 691-706. doi:10.1007/s10988008-9030-5.

Willer, Malte \& Christopher Kennedy. 2020. Assertion, expression, experience. Inquiry doi:10.1080/0020174X.2020.1850338.

Wyatt, Jeremy. 2021. The nature of disagreement: matters of taste and environs. Synthese doi:10.1007/s11229-021-03266-6.

Zakkou, Julia. 2019. Faultless Disagreement: A defense of contetualism in the realm of personal taste. Verlag Vittorio Klostermann.

Deniz Rudin

3601 Watt Way, GFS 301

University of Southern California

Los Angeles, CA 90089

drudin@usc.edu
Elsi Kaiser

3601 Watt Way, GFS 301

University of Southern California

Los Angeles, CA 90089

emkaiser@usc.edu 